\title{
Atom-Probe Tomography Measurements of Isotopic Ratios of High-field Materials with Corrections and Standardization: a Case Study of the ${ }^{12} \mathrm{C} /{ }^{13} \mathrm{C}$ of Meteoritic Nanodiamonds
}

\author{
J. B. Lewis ${ }^{1}$, D. Isheim ${ }^{2}$, C. Floss ${ }^{1}$, T. L. Daulton ${ }^{1,3}$, D. N. Seidman ${ }^{2}$ \\ ${ }^{1 .}$ Laboratory for Space Sciences, Physics Department, Washington University, St. Louis, MO, USA. \\ 2. Center for Atom-Probe Tomography, and Dept. of Materials Science and Engineering, Northwestern University, \\ Evanston, IL, USA. \\ ${ }^{3 .}$ Institute of Materials Science and Engineering, Washington University, St. Louis, MO, USA.
}

Efforts are ongoing to measure the ${ }^{12} \mathrm{C} /{ }^{13} \mathrm{C}$ ratios of individual $(\sim 2.6 \mathrm{~nm}$ diameter) meteoritic nanodiamond (ND) grains using atom-probe tomography (APT) [1]. The origins of meteoritic NDs remain an enigma, which could be resolved by isotopic measurements of individual ND grains. Isotopic anomalies in trace elements, chiefly Xe in bulk meteoritic acid dissolution residues [2], suggest a Type II supernova origin for at least a subpopulation of the NDs. While the bulk measured C [3] and N [4] are consistent with formation in the Solar System, bulk measurements may not be diagnostic. Poorly-crystalline to amorphous carbonaceous phases, which can comprise a significant fraction of the residue [5], are potentially of different origin than the NDs and hence can have different C isotopic compositions. Measurements of NDs by APT is challenging because C, being a "high field" element, is known to yield biased isotopic ratios prior to correction $[1,6]$. We outline an analysis procedure to calculate isotopic ratios for $\mathrm{C}$, employed on an ensemble of detonation synthesized ND standards (DND) and meteoritic ND residue from the Allende DM separate (ADM).

A full description of specimen preparation is given in [1,6]. After focused ion-beam microscope liftout, APT of NDs embedded between sputter-deposited Pt layers was performed with a Cameca LEAP 4000X Si [1, 6], yielding 3D positions and time-of-flight data for $\sim 57 \%$ of the atoms (i.e., collection yield) in needle-shaped sample "nanotips" of radius $20-100 \mathrm{~nm}$. We use laser pulsing to reduce the probability of nanotip fracture; however, higher laser pulse fractions lead to more delayed evaporation events, which manifest themselves as peak-tails. Since isotopes of the same element generally have similar mass-to-charge-state $(\mathrm{m} / \mathrm{n})$ ratios, they are particularly susceptible to tail interferences. Peak shape is determined by the base temperature, length of the laserinduced thermal pulse, nanotip shape, and material properties. Unfortunately, no standardized peak-fitting method exists for APT. In the procedure we developed, the background is calculated by a linear fit to the baseline of the time-of-flight spectrum and subtracted. We then define the mass range about a given major peak as the full width at half maximum (FWHM). The bin size is set so that the defined range is five bins wide. For minor isotopes we use the same range width as used for the major isotope, rather than FWHM; e.g., ${ }^{13} \mathrm{C}^{+}$is fit with the same range width as ${ }^{12} \mathrm{C}^{+}$. This is because for same-element, same-charge-state ions, none of the factors affecting peak shape should vary, and the shift in time-of-flight/amu over $1 \mathrm{amu}$ is negligibly small compared to bin size. Therefore, ranges are better defined by the largest peaks. Finally, for ${ }^{13} \mathrm{C}(+\&++)$ the contribution from the tails of ${ }^{12} \mathrm{C}(+\&$ $++)$ is estimated and subtracted. This procedure is largely based on [7].

Mean standard ratios are calculated along with standard error of the mean $\left(\sigma_{\mathrm{M}}\right)$ and standard deviation. The standard data are compared to Allende data in Fig. 1. $\sigma_{\mathrm{M}}$ is representative of the uncertainty in the determination of the mean value, while the standard deviation represents the scatter of the data. The standards reproduce the significant instrumental artifacts, noted in prior work, which lead to an underestimation of ${ }^{12} \mathrm{C} /{ }^{13} \mathrm{C}$ ratios $[1,6]$.

The mean Allende ratio (for NDs and poorly-crystalline to amorphous $\mathrm{C}$ residuals) is, within uncertainties, the same as that of the mean standard ratio. The scatter in the ADMs is not significantly greater than for the standards, consistent with isotopically normal grains. We conclude that little if any of the carbonaceous material we have analyzed from the Allende residue is presolar. Given our current sample size, the presence of nondiamond carbonaceous material, and remaining instrumental artifacts, this conclusion should not be applied too 
broadly. The mean ratio of ${ }^{12} \mathrm{C} /{ }^{13} \mathrm{C}$ in charge state + is significantly greater than in charge state ++ . There is no reason to believe the different charge states of $\mathrm{C}$ would have different isotopic ratios; therefore, we take this difference to be the result of an experimental artifact that increases the measured ${ }^{12} \mathrm{C}^{+}$and/or ${ }^{13} \mathrm{C}^{++}$as well as possibly decreasing the measured ${ }^{12} \mathrm{C}^{++}$and/or ${ }^{13} \mathrm{C}^{+} .\left({ }^{12} \mathrm{CH}\right)$ hydride interference would give the opposite effect.

If ${ }^{12} \mathrm{C}^{++}$is preferentially affected by deadtime effects compared to ${ }^{12} \mathrm{C}^{+}$, it could explain the discrepancy, as could a ${ }^{12} \mathrm{C}_{2}{ }^{++}$interference at $12 \mathrm{amu}$. Iterative proportional fitting of detected multiple isotope pairs [8], or Poisson statistics [9] can be used to generate a correction for signal loss due to detector deadtime, providing there are enough counts. To date, however, such methods have generated only small changes to the measured ratios. On average the deadtime correction to the ${ }^{12} \mathrm{C}^{++}$peak is several orders of magnitude greater than to the ${ }^{12} \mathrm{C}^{+}$peak.

We intend to survey available APT ND data to determine if undercorrected deadtime signal loss is responsible for the low measured standard ratios, and to assess the effect of ${ }^{12} \mathrm{C}_{2}^{++}$and $\left({ }^{12} \mathrm{CH}\right)^{+}$on $\mathrm{C}$-ratio measurements. Correlated transmission electron microscopy and APT of nanotips will allow us to compare the crystal structure of the $\mathrm{C}$ residue with the reconstructed $\mathrm{m} / \mathrm{n}$ data, and allow us to distinguish between $\mathrm{ND}$ and amorphous $\mathrm{C}$ phases.

\section{References:}

[1] Heck P. R. et al, Meteoritics and Planetary Science 49 (3) (2014), pp. 453-467.

[2] Lewis R. S. et al, Nature 326 (1987), pp. 160-162.

[3] Russell S. R. et al, Meteoritics and Planetary Science 31 (1996), pp. 343-355.

[4] Marty B. et al, Science 332 (2011), pp. 1533-1536.

[5] Stroud R. M. et al, Astrophysical Journal Letters 738 (2011), L27-L31.

[6] Isheim D. et al, Microscopy and Microanalysis 19 (Suppl 2) (2013), CD974-CD975.

[7] Hudson D. et al, Ultramicroscopy 111 (2011), pp. 480-486.

[8] Lewis J. B. et al, $46^{\text {th }}$ Lunar and Planetary Science Conference (2015), \#1480.

[9] Stephan T. et al, International Journal of Mass Spectrometry (2015, in press).

[10] Coplen T. B. et al, Pure and Applied Chemistry 74 (10) (2002), pp. 1987-2017.

[11] This work is supported by NASA grants NNX14AP15H (J.B.L.) and NNX13AF53G (C.F.). The atom-probe tomograph at the Northwestern University Center for Atom-Probe Tomography (NUCAPT) was acquired with support from NSF and ONR.

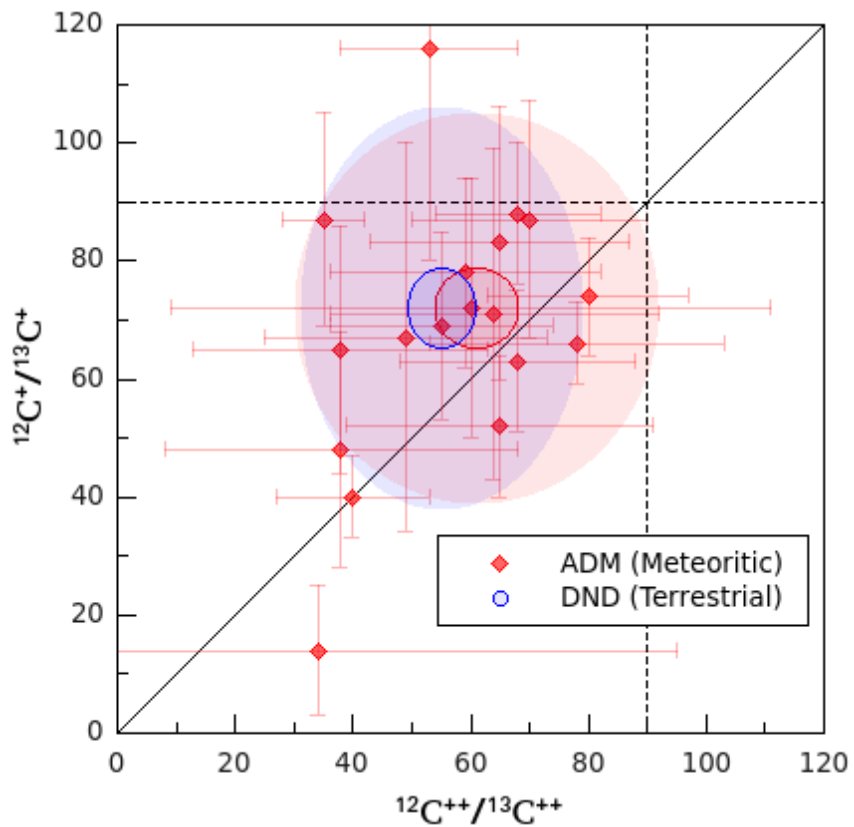

Fig. 1 Mean C isotopic ratios for 24 terrestrial detonation ND standards and 21 Allende DM ND data sets. 18 of the Allende measurements had sufficient ${ }^{13} \mathrm{C}^{++}$counts for ratio calculations; each of these is plotted as a diamond symbol with $\pm 2 \sigma$ error bars. Small ovals represent $\pm 2 \times$ the standard error of the mean. Large ovals represent $\pm 2 x$ the standard deviation of a datum from the mean. The diagonal line is the line of equal ratio for isotopes at the two different charge states. The horizontal and vertical lines are the expected Solar System isotopic ${ }^{12} \mathrm{C} /{ }^{13} \mathrm{C}$ ratio, $\sim 89$ [10]. 